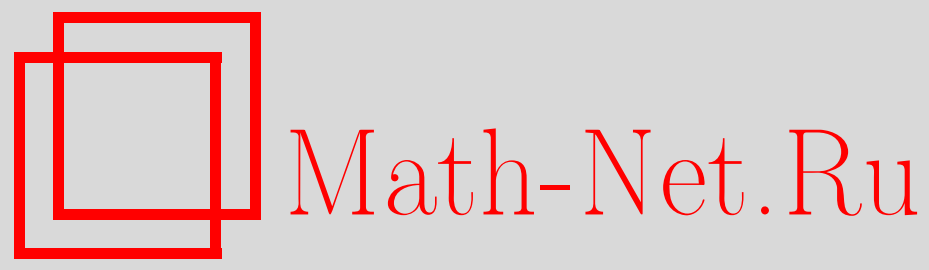

В. А. Рыжов, О сингулярном и абсолютно непрерывном подпространствах несамосопряженного оператора, характеристическая функция которого обладает граничными значениями на вещественной оси, Функи. анализ и его прил., 1998, том 32, выпуск 3, 83-87

DOI: https://doi.org/10.4213/faa432

Использование Общероссийского математического портала Math$\mathrm{Net.Ru}$ подразумевает, что вы прочитали и согласны с пользовательским соглашением

http://www . mathnet.ru/rus/agreement

Параметры загрузки:

IP : 18.208 .226 .222

26 апреля 2023 г., 15:58:26

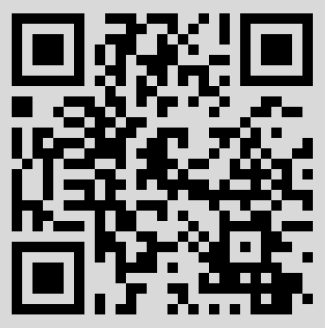




\title{
О сингулярном и абсолютно непрерывном подпространствах несамосопряженного оператора, характеристическая функция которого обладает граничными значениями на вещественной оси
}

\author{
(c) 1998. В. А. Рыжов
}

Целью заметки является изложение ряда результатов, связанных с внутренней структурой абсолютно непрерывных и сингулярных подпространств несамосопряженного оператора. По своему направлению она примыкает к исследованиям [1-7], распространяя часть полученных там результатов на более широкий класс операторов и формулируя эти результаты в виде прямых обобщений известных положений «самосопряженной» теории.

Задачи несамосопряженной теории рассеяния, рассматриваемые в контексте общей спектральной теории линейных операторов, оформились в самостоятельный раздел под значительным влиянием работ Т. Като, развившего в [15] теорию гладких возмущений операторов, до сих пор активно используемую при изучении вопросов устойчивости абсолютно непрерывного спектра [13], а также работ Л. А. Сахновича $[16,17]$, основным объектом исследования которых является характеристическая оператор-функция несамосопряженного оператора, введенная М. С. Лившицем [18] и ставшая к настоящему времени предметом многочисленных исследований (см., например, $[2,10,11])$. Последовавшее вслед за этим развитие подхода функциональной модели в теории несамосопряженных операторов, существенно опирающееся на понятие характеристической функции, принесло ряд новых важных результатов, касающихся как устойчивости спектра в смысле теории возмущений, так и собственно построения «несамосопряженной» теории рассеяния $[1-9,11]$. Настоящая заметка содержит результаты о строении (локальных) абсолютно непрерывного и сингулярного подпространств линейного несамосопряженного оператора общего вида, характеристическая операторфункция которого обладает граничными значениями почти всюду на вещественной оси. Тем самым ряд положений работ [1-7] распространяется сразу в двух направлениях: первое - ослабляется требование существования скалярного кратного у характеристической функции оператора, гарантирующее существование указанных граничных значений, и второе - рассматриваемый оператор может не допускать представления вида «самосопряженный + возмушение», чем охватывается ситуация «несамосопряженного граничного условия», возникающая в теории граничных задач для дифференциальных операторов. Помимо этого работа содержит и представляющиеся новыми сведения по вопросам описания (локальных) абсолютно непрерывного и сингулярного подпространств несамосопряженного оператора. Доказательства всех полученных результатов существенно опираются на технику функциональной модели, однако приводимые здесь формулировки не используют «модельной» терминологии. 
В сепарабельном гильбертовом пространстве $H$ рассматривается замкнутый оператор $L$ с плотной в $H$ областью определения $D(L)$. Предполагается, что $\rho(L) \cap \mathbb{C}_{ \pm} \neq \varnothing(\rho(L)$ - резольвентное множество оператора $L$, $\left.\mathbb{C}_{ \pm}:=\{z \in \mathbb{C} \mid \pm \operatorname{Im} z>0\}\right)$ и, кроме того, $L$ вполне несамосопряжен. Последнее означает отсутствие у $L$ нетривиальных приводящих самосопряженных частей ${ }^{1}$. Напомним определение характеристической функции оператора $L$ (см. [10] или [11]). Пусть для определенности $(-i) \in \rho(L)$. Операторы $Q:=R+R^{*}-2 R^{*} R, Q_{*}:=R+R^{*}-2 R R^{*}$, где $R:=(L+i)^{-1}$, являются самосопряженными в $H$ и $J:=\left.\operatorname{sign} Q\right|_{E}, J_{*}:=\left.\operatorname{sign} Q_{*}\right|_{E_{*}}$, где $E:=\cos Q H, E_{*}:=\cos Q_{*} H$, являются инволюциями в $E, E_{*}$ соответственно. Нормированная характеристическая оператор-функция $\Theta(\cdot)$ оператора $L$ аналитична на $\rho\left(L^{*}\right)$, принимает значения в алгебре ограниченных операторов, действующих из $E$ в $E_{*}$ и однозначно определяется тождеством $\Theta(z) G u=G_{*}\left(L^{*}-z\right)^{-1}(L-z) u\left(u \in D(L), z \in \rho\left(L^{*}\right)\right)$. Здесь $G$, $G_{*}$ - замыкания отображений $\widetilde{G}:=|Q|^{1 / 2}(L+i), \widetilde{G}_{*}:=\left|Q_{*}\right|^{1 / 2}\left(L^{*}-i\right)$, заданных на $D(G) \equiv D(L)$ и $D\left(G_{*}\right) \equiv D\left(L^{*}\right)$ соответственно. Заметим, что $(-i)[(L u, v)-(u, L v)]=(J G u, G v)$ для $u, v \in D(L)$ (и аналогичное равенство справедливо с заменой $L, J, G$ на $-L^{*}, J_{*}, G_{*}$ для $\left.u, v \in D\left(L^{*}\right)\right)$.

Всюду ниже считается выполненным следующее

Основное ПРЕДПоложениЕ. Найдется такая почти всюду ${ }^{2}$ положительная функция $\varepsilon^{0}(k), k \in \mathbb{R}$, что для почти всех $k \in \mathbb{R}$ существует

$$
\mathrm{w}-\lim _{\varepsilon \downarrow 0} \Theta(k \pm i \varepsilon)=: \Theta(k \pm i 0), \quad \varepsilon<\varepsilon^{0}(k) .
$$

Из результатов работы [10] легко следует, что основное предположение выполнено (или нет) для операторов $L$ и $L^{*}$ одновременно.

Ниже $R(z):=(L-z I)^{-1}, z \in \rho(L),-$ резольвента оператора $L, \delta \subset \mathbb{R}-$ измеримое подмножество вещественной оси $\mathbb{R}$ и $\chi_{\delta}(\cdot)$ - его индикатор.

ПреДЛОЖЕНИЕ 1. Для $u, v \in H$ существуют при п.в. $k \in \mathbb{R}$ предельь

$$
\mathrm{w}-\lim _{\varepsilon \downarrow 0}[G R(k \pm i \varepsilon) u], \quad \lim _{\varepsilon \downarrow 0} \mathscr{F}_{u, v}^{ \pm}(k \pm i \varepsilon),
$$

где $\mathscr{F}_{u, v}^{ \pm}(z):=(R(z) u, v), z \in \rho(L) \cap \mathbb{C}_{ \pm}$(cм. [5]).

Пусть $\partial \mathscr{F}_{u, v}(k):=\mathscr{F}_{u, v}^{+}(k+i 0)-\mathscr{F}_{u, v}^{-}(k-i 0)$ для п.в. $k \in \mathbb{R}$,

$$
\begin{aligned}
N_{s}(\delta) & :=\left\{u \in H \mid \partial \mathscr{F}_{u, v}(k)=0 \text { для п.в. } k \in \delta, \forall v \in H\right\}, \\
N_{* s}(\delta) & :=\left\{v \in H \mid \partial \mathscr{F}_{u, v}(k)=0 \text { для п.в. } k \in \delta, \forall u \in H\right\}, \\
N_{a}(\delta) & :=H \ominus N_{* s}(\delta) .
\end{aligned}
$$

ОПРеДЕлЕниЕ 1 . Для $\delta \subset \mathbb{R}$ подпространства $N_{s}(\delta), N_{a}(\delta)$ называются (локальными) сингулярным и абсолютно непрерывным подпространствами оператора $L$, соответствующими множеству $\delta$.

${ }^{1}$ Это предположение по существу не является ограничением.

${ }^{2}$ Понятия «почти всюду», «почти все» (п.в.) и понятие измеримости относятся к мере Лебега на вещественной оси $\mathbb{R}$. 
ПРЕДЛОЖЕНИЕ 2. $N_{s}(\delta), N_{a}(\delta)$ ультраинвариантньл [11] относительно $L$. Ниже приводятся более явные описания подпространств $N_{s}(\delta), N_{a}(\delta)$.

ТеоремА 1. Для $u \in H$ следуюшие утверждения эквивалентнь:

1) $u \in N_{s}(\delta)$;

2) $G R(k+i 0) u=G R(k-i 0) u$ для n. в. $k \in \delta$, zде $G R(k \pm i 0) u:=$ $\mathrm{w}-\lim _{\varepsilon \downarrow 0}[G R(k \pm i \varepsilon) u]$.

Если в дополнение $\kappa$ основному предположению для $n . в . k \in \mathbb{R}$ сущеcтвуют $\mathrm{s}^{-} \lim _{\varepsilon \downarrow 0}[\Theta(k \pm i \varepsilon)]^{*}$, то любое из утверждений 1), 2) равносильно каждому из следуюших четьрех:

$\left.3^{ \pm}\right) \lim _{\varepsilon \downarrow 0} \varepsilon\|R(k \pm i \varepsilon) u\|^{2}=0$ для n. в. $k \in \delta ;$

$\left.4^{ \pm}\right) \alpha^{ \pm}(u, v ; k)=0$ для n. в. $k \in \delta, v \in H$, где

$$
\alpha^{ \pm}(u, v ; k):=\lim _{\varepsilon \downarrow 0} \varepsilon(R(k \pm i \varepsilon) u, R(k \pm i \varepsilon) v),
$$

причем указанные предель существуют для $u, v \in H$ при $n$.в. $k \in \mathbb{R}$.

TEOPEMA 2. 1. $N_{a}(\delta)=\operatorname{clos} \widetilde{\mathscr{N}}(\delta)=\operatorname{clos} \widetilde{\mathscr{N}}_{w}(\delta)$, ade

$$
\begin{aligned}
\widetilde{\mathscr{N}}(\delta):=\{u \in H \mid & G R(z) u \in H_{2}^{ \pm}(E), \\
& G R(k+i 0) u=G R(k-i 0) u \text { для n. в. } k \in \mathbb{R} \backslash \delta\},
\end{aligned}
$$$$
\widetilde{\mathscr{N}}_{w}(\delta):=\left\{u \in H \mid \mathscr{F}_{u, v}^{ \pm} \in H_{2}^{ \pm}, \partial \mathscr{F}_{u, v}(k)=0 \text { для n. в. } k \in \mathbb{R} \backslash \delta, \forall v \in H\right\}
$$

$\left(H_{2}^{ \pm}, H_{2}^{ \pm}(E)\right.$ - классь Xарди скалярньлх и Е-значньх функиий).

2. Для измеримого множества $X, X \subset \mathbb{R}$, обозначим через $H_{X}$ максимальное инвариантное подпространство оператора $L$, такое, ито $\left.L\right|_{H_{X}}$ подобен самосопряженному абсолютно непрерьвному оператору со спектром в $X\left(H_{X}=\{0\}\right.$, если такого пространства не существует). Тогда $H_{X} \subset N_{a}(X) u N_{a}(X)=\operatorname{span}\left(H_{X} \mid X \subset \delta\right)$.

ТеоремА 3. Для $u \in H$ следуюшие утверждения 1)-3) равносильнь:

1) $u \in \widetilde{\mathscr{N}}_{w}(\delta)$;

2) $u \in \widetilde{\mathscr{N}}_{w}(\delta)$ и найдетсл константа $C=C(u)<\infty$, такая, ито $\left\|\mathscr{F}_{u, v}^{ \pm}\right\|_{L_{2}}$ $\leqslant C\|v\|$ npu всеx $v \in H$;

3) для любого оператора $B: H \rightarrow H$ класса Гильберта-Шмидта $B R(z) u$ $\in H_{2}^{ \pm}(H)$ u $B R(k+i 0) u=B R(k-i 0) u$ npu n. в. $k \in \mathbb{R} \backslash \delta$, zде $B R(k \pm i 0) u:=$ $\lim _{\varepsilon \downarrow 0}[B R(k \pm i \varepsilon) u]$ существует для $n$. в. $k \in \mathbb{R}[2,11]$.

Отметим, что при более сильных, нежели основное предположение, ограничениях, случай $\delta=\mathbb{R}$ теоремы 3 и равенство $\operatorname{clos} \widetilde{\mathscr{N}}_{w}(\mathbb{R})=N_{a}(\mathbb{R})$ из теоремы 2 доказаны в [4].

Изложенное выше позволяет ввести функциональное исчисление для абсолютно непрерывной части оператора $L$ (т.е. для сужения $\left.L\right|_{N_{a}(\mathbb{R})}$ ) «привычным» способом и проверить его согласованность с имеющимся $[1,4,5]$. Приведем лишь один относящийся сюда результат.

TEOpema 4. Пусть $\delta \subset \mathbb{R}$. Тогда $\partial \mathscr{F}_{u, v} \in L_{1}(\delta)$ для всех $u \in \widetilde{H}(\delta):=$ $\widetilde{\mathscr{N}}(\mathbb{R})+N_{s}(\delta), v \in H$ и оператор $\mathscr{P}(\delta)$, определенный на $\widetilde{H}(\delta)$ своей формой 
$(\mathscr{P}(\delta) u, v):=(2 \pi i)^{-1} \int_{\delta} \partial_{\mathscr{F}_{u, v}}(k) d k, u \in \widetilde{H}(\delta), v \in H$, является спектральнымм проектором из $\widetilde{H}(\delta)$ на $\widetilde{\mathscr{N}}(\delta)$ параллельно $N_{s}(\delta)$ в смысле выполнения следуюших равенств:

$$
\begin{gathered}
\mathscr{P}(\delta) \widetilde{H}(\delta)=\widetilde{\mathscr{N}}(\delta), \quad \operatorname{ker} \mathscr{P}(\delta)=N_{s}(\delta), \quad[\mathscr{P}(\delta)]^{2}=\mathscr{P}(\delta), \\
R(z) \mathscr{P}(\delta) u=\mathscr{P}(\delta) R(z) u, \quad u \in \widetilde{H}(\delta), \quad z \in \rho(L) .
\end{gathered}
$$

Кроме того, $\mathscr{P}(\delta) u=\chi_{\delta}\left(\left.L\right|_{N_{a}(\mathbb{R})}\right) u$ для $u \in \widetilde{H}(\delta)$, где правал часть определена с помощью функциональной модели $L[1,4,5]$.

Следующая теорема является «локальным» обобщением некоторых результатов из $[2,3,7]$ на рассматриваемый здесь случай.

Теорема 5. Пусть для $\delta \in \mathbb{R}$ имеет место оченка $(C(\delta)<\infty)$

$\operatorname{ess} \sup \left(\left\|\left(I+J_{*}\right) \Theta(k-i 0)(I+J)\right\|+\left\|\left(I-J_{*}\right) \Theta(k+i 0)(I-J)\right\|\right) \leqslant C(\delta)$. $k \in \delta$

Тогда

1) $\|\mathscr{P}(\delta)\|<\infty$

2) $N_{a}(\delta) \cap N_{s}(\delta)=\{0\}$

3) $\widetilde{\mathscr{N}}(\delta)=N_{a}(\delta)$;

4) $N_{a}(\delta) \dot{+} N_{s}(\delta)=H$;

5) $N_{a}(\delta)=H_{\delta}$ (см. теорему 2).

ПримеР. В качестве простого иллюстративного примера несамосопряженного оператора, для которого выполнено основное предположение, рассмотрим оператор Шрёдингера $\mathscr{L}$, определенный как псевдорасширение по Фридрихсу [12] заданной на $C_{0}^{\infty}\left(\mathbb{R}^{n}\right)$ суммы $-\Delta+V(x)$, где $-\Delta-$ свободный Лапласиан в $H:=L_{2}\left(\mathbb{R}^{n}\right)$, а комплекснозначная функция $V(\cdot)$ такова, что $\left(1+|x|^{2}\right)^{1 / 2+\gamma} V(x)(-\Delta+I)^{-1}$ компактен $($ в $H)$ для некоторого $\gamma>0$ $(V$ - комплексный потенциал Агмона [13]). Характеристическая функция $\Theta(z)$ вполне несамосопряженной части оператора $\mathscr{L}$ определена при всех $z \in \mathbb{C}$ с достаточно большой мнимой частью; она может быть записана в виде

$$
\Theta(z)=\left.\left(I+2 i\left|V_{I}\right|^{1 / 2}\left(\mathscr{L}^{*}-z I\right)^{-1}\left|V_{I}\right|^{1 / 2} J\right)\right|_{E}, \quad z \in \rho\left(\mathscr{L}^{*}\right),
$$

где $V_{I}:=(2 i)^{-1}\left(V-V^{*}\right), J:=\operatorname{sign} V_{I}, E:=\operatorname{clos} V_{I} H$, см. [8, 14]. Для нее

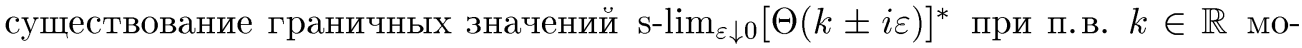
жет быть проверено аналогично [9]. Несложно также было бы вывести этот результат из следующего варианта принципа предельного поглощения для оператора $\mathscr{L}$ (ср. [13]):

Tеорема. Пусть $H_{t}:=\left\{\left.f(x)\left|\|f\|_{t}^{2}:=\int_{\mathbb{R}}\left(1+|x|^{2}\right)^{t}\right| f(x)\right|^{2} d x<\infty\right\}, t \in \mathbb{R}$. При $t>1 / 2$ существуют предельь $\lim _{\varepsilon \downarrow 0}(\mathscr{L}-k \pm i \varepsilon)^{-1}$ для n.в. $k \in \mathbb{R}$ по норме алгебры ограниченных операторов, действующих из $H_{t}$ в $H_{-t}$.

Другие примеры операторов ${ }^{3}$, для которых выполнено основное предположение настоящей работы, легко можно построить, опираясь на следующее

ЗЗаметим, что, вообще говоря, сформулированное ниже утверждение неприменимо к только что приведенному в качестве примера оператору Шрёдингера. 
(доказанное в [5]) утверждение:

УТВЕРЖДЕНИЕ. Пусть разность $(L-z I)^{-1}-(A-z I)^{-1}$ ядерна для некоторого $z \in \rho(L) \cap \rho(A)$, где $A=A^{*}$ - самосопряженный оператор в $H$. Тогда предель $\mathrm{s}-\lim _{\varepsilon \downarrow 0} \Theta(k \pm i \varepsilon),{ }_{\mathrm{s}}-\lim _{\varepsilon \downarrow 0}[\Theta(k \pm i \varepsilon)]^{*}$ существуют для n.в. $k \in \mathbb{R}($ здесь $\Theta(\cdot)$ - характеристическая функция вполне несамосопряженной части оператора $L$ ).

В заключение автор благодарит С. Н. Набоко за внимание к работе.

\section{ЛИТЕРАТУРА}

1. Набоко С. Н. Зап. научн. семинаров ЛОМИ, 65, 90-102 (1976). 2. Павлов Б. С. Изв. АН СССР, сер. матем., 39, 123-148 (1975). 3. Веселов В. Ф. Вестн. ЛГУ, мат., мех., астр., 2, 11-17 (1988). 4. Тихонов A. C. Абсолютно непрерывный спектр линейного оператора. УкрНИИНТИ, Симферополь, 2471-Ук88, 1988. 5. Makarov N. G., Vasjunin V. I. In: Lecture Notes in Math., Vol. 864, 1981, pp. 365-412. 6. Makarov N. G. In: 49 Alfred Haar Memorial Conference, 1985, pp. 611-621. 7. Набоко С. Н. Зап. научн. семинаров ЛОМИ, 113, 149-177 (1981). 8. Набоко С. Н. Труды МИАН, 147, 86-114 (1980). 9. Набоко C. Н. В кн.: Пробл. мат. физики, вып. 12, Изд-во ЛГУ, Л., 1987, с. 132-135. 10. Шmpayc А. В. Изв АН СССР, сер. матем., 24, вып. 1, 4374 (1960). 11. Секефальви-Надь Б., Фояш Ч. Гармонический анализ операторов в гильбертовом пространстве. Мир, М., 1970. 12. Kamo T. Теория возмущений линейных операторов. Мир, М., 1972. 13. Рид М., Саймон Б. Методы современной математической физики, т. 4: Анализ операторов. Мир, М., 1982. 14. Павлов Б. С. Матем. сб., 102, вып. 4, 511-526 (1977). 15. Kato T. Math. Ann., 162, 258-279 (1966). 16. Сахнович Л. А. Труды МMO, 19, 211-270 (1968). 17. Сахнович Л. А. Функц. анализ и его прил., 2, вып. 1, 51-63 (1968). 18. Ливиии М. С. Матем. сб., 34, вып. 1, 145-199 (1954).

Санкт-Петербургская государственная академия аэрокосмического приборостроения
Поступило в редакцию

2 июля 1993 г.

В переработанном виде 18 декабря 1997 г.

\section{K вопросу о классификации банаховых алгебр сингулярных интегральных операторов с $P C$-коэффициентами в пространствах $L_{p}$ на сложных контурах}

(c) 1998. В. А. Стукопин

1. В данной работе уточняется теорема о классификации банаховых алгебр сингулярных интегральных операторов с $P C$-коэффициентами, действующих в $L_{p}$-пространствах на сложных контурах, в случае заранее не фиксированного показателя $p$. В частном случае, когда $p$ фиксировано, эта теорема была сформулирована в [1] (теорема 5). 